\title{
Enumerating Large Orbits and Direct Condensation
}

\author{
Frank Lübeck and Max Neunhöffer
}

\section{CONTENTS}

1. Introduction

2. The Orbit Algorithm and Variations

3. Matrices Acting on Vectors

4. Parallelization

5. Direct Condensation

Acknowledgements

Electronic Availability

References
We describe a new algorithm for direct condensation, which is a tool in computational representation theory. The crucial point for this is the enumeration of very large orbits for a group acting on some set. We present a variation of the standard orbit enumeration algorithm that reduces the amount of storage needed and behaves well under parallelization. For the special case of matrices acting on a finite vector space an efficient implementation is described. This allows us to use condensation methods for considerably larger permutation representations than could be handled before.

\section{INTRODUCTION}

Notation. Let $G=\left\langle g_{1}, \ldots, g_{r}\right\rangle$ be a group given by $r$ generators. Let $M$ be a set, $\operatorname{Sym} M$ the symmetric group on $M$ and $\pi: G \rightarrow \operatorname{Sym} M$ a homomorphism. We say that $G$ acts on $M$, and for $m \in M$ and $g \in G$ we write $m g:=\pi(g)(m)$. Clearly, $\pi$ is uniquely determined by the images $\pi\left(g_{i}\right), 1 \leq i \leq r$, of its generators. We call the elements of $M$ points, and for $m \in M$ the set $m G:=\{m g \mid g \in G\}$ is called the $G$-orbit of $m$ (under the action $\pi$ ). Let $m_{0} \in M$ such that its $G$-orbit $m_{0} G$ is finite.

Let $K$ be a subgroup of $G$, also given by a finite number of generators. The main purpose of this paper is the discussion of an algorithm which computes for each $K$-orbit $m K \subset m_{0} G$, where $m \in m_{0} G$, the intersection numbers of its translates $m K g_{i}$ with all such $K$-orbits. This is explained in Section 2 which starts with the description of two basic algorithms for enumerating an orbit.

An interesting interpretation of these numbers in the representation theory of the group $G$ is explained briefly in Section 5, which also contains two explicit examples. This application, called direct condensation, was our original motivation for this note. But we hope that our general remarks about enumerating large orbits will be useful for other applications as well. 
The result in Section $5 \mathrm{C}$ is of independent interest because it can be used to finish the determination of the (previously unknown) decomposition numbers of the symmetric groups $S_{n}$, for $21 \leq n \leq 23$, in characteristic 5 .

Our algorithm for orbit intersection matrices (Algorithm 2.5) is given in such a way as to make it easy to find variations allowing practical application to very large cases. For example, it may not be possible to store all points of the orbit on a computer for lack of memory. Therefore we introduce a concept of minimal points in an orbit and only such points need to be stored during the algorithm.

In Section 3 we make this explicit in the important practical case were $G$ acts via matrices on a (finite) vector space. We show a way to define minimal elements that allows us to get very efficiently from an arbitrary vector in the orbit to a minimal one in the same $K$-orbit. This is the key point which makes our algorithm for direct condensation much more efficient than a previous implementation described in [Cooperman and Tselman 1996] (see Section 5B for more details). In Section 4 we discuss a parallelization of the algorithm and our implementation. This can be used to treat substantially larger cases than could be handled before.

\section{THE ORBIT ALGORITHM AND VARIATIONS}

In this section we first describe algorithms to enumerate the $G$-orbit $m_{0} G$ from the given $m_{0} \in M$ and given $\pi\left(g_{i}\right)$, where $1 \leq i \leq r$. (Recall that we assume $m_{0} G$ finite.)

\section{A. The Basic Algorithm}

\section{Algorithm 2.1 (Orbit)}

Input: $m_{0} \in M$ and $\pi\left(g_{i}\right)$ for $1 \leq i \leq r$

Output: a list $L$ containing the elements of $m_{0} G$

Initialize: $L \leftarrow\left[m_{0}\right]$

for $m$ in $L$

for $i$ from 1 to $r$

$x \leftarrow m g_{i}$

if $x \notin L$

append $x$ to the list $L$

return $L$

The algorithm terminates because the orbit $m_{0} G$ is finite. It is clear that the resulting list $L$ contains only elements from the $G$-orbit of $m_{0}$. Furthermore
$L$ is invariant under the action of the generators $g_{i}$ and so under their inverses $g_{i}^{-1}$. Since each element of $G$ is a finite product of these generators and their inverses, $L$ is invariant under the action of $G$. This shows that $L$ contains exactly the elements of the orbit $m_{0} G$.

Counting the number of necessary operations in the algorithm we find:

Proposition 2.2. Algorithm 2.1 needs $r$ times the length of $m_{0} G$ operations consisting of an application of a generator of $G$ to a point and a lookup of the resulting point in the list of known points.

With a naïve implementation of the lookup of points in $L$ by sequential comparison, the lookup part of an operation described in the proposition would take most of the running time in the case of large orbits. But with the help of standard techniques like hashing [Knuth 1997, Section 6.4], the time for a single lookup becomes (almost) independent of the length of the orbit.

\section{B. Large Orbits}

Our interest here is the practical enumeration of large orbits. Two problems arise: the list of all points in an orbit does not fit into the computer memory and the running time of the algorithm may be longer than we want to wait for the result.

Both problems are addressed by the following variation of Algorithm 2.1.

We now assume that we have a partition of $M$ that is a refinement of the partition into $G$-orbits. As a typical example think of the partition into orbits under a subgroup of $G$. Furthermore we assume that for each part a nonempty subset is defined whose points we will call minimal. Finally we assume the availability of three functions (whose implementation is discussed later):

- part, which returns for a given $m \in M$ a list of the points in the part containing $m$.

- minimals, which returns for a given $m \in M$ a list of the minimal points of part $(m)$.

- minimal, which returns for a given $m \in M$ one minimal point in $\operatorname{part}(m)$.

To save memory for the orbit $m_{0} G$ the following algorithm computes only a list of its minimal points. 
Algorithm 2.3 (OrbitByPartition)

Input: $m_{0} \in M$ and $\pi\left(g_{i}\right)$ for $1 \leq i \leq r$

Output: a list $L$ containing the minimal elements of $m_{0} G$ with one element of each part marked as a representative

Initialize: $L \leftarrow \operatorname{minimals}\left(m_{0}\right)$; mark first element as a representative

for $m^{\prime}$ in $L$ which is marked as a representative

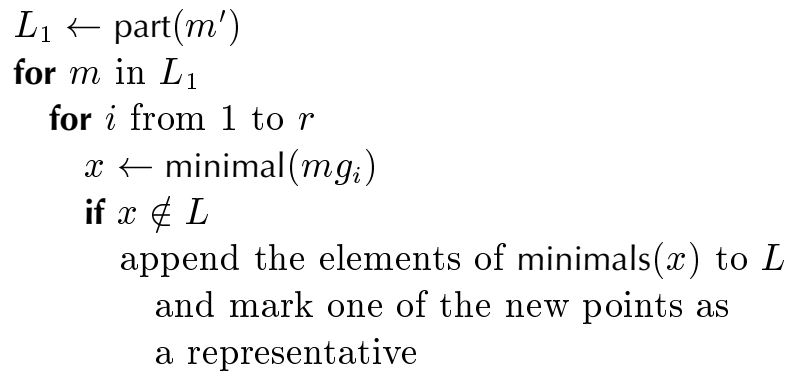

return $L$

With the output $L$ of this algorithm it is possible to run through all points in $m_{0} G$ using the function part as above. Also, one can check for an arbitrary point $m \in M$ whether it is contained in $m_{0} G$ by checking whether minimal $(m)$ is in $L$.

The order in which the parts are handled in the outer loop of this algorithm does not matter (except for the ordering of the points in the resulting list $L)$. We will show in Section 4 how to use this for parallelizing the algorithm.

\section{C. Orbit Intersection Matrices}

Let $K=\left\langle k_{1}, \ldots, k_{s}\right\rangle$ be a subgroup of $G$ given by $s$ generators and let $m_{1}, \ldots, m_{m}$ be representatives of the $K$-orbits within $m_{0} G$.

Definition 2.4. In this setting we define the $K$-orbit intersection matrices of the $g_{i}$ on $m_{0} G$ to be the matrices $\left(a_{k l}\left(g_{i}\right)\right)_{1 \leq k, l \leq m}$ with

$$
a_{k l}\left(g_{i}\right):=\left|m_{k} K g_{i} \cap m_{l} K\right| \text { for } 1 \leq i \leq r .
$$

We are interested in the practical computation of these orbit intersection matrices. In Section 5 we will discuss an application of these matrices in the representation theory of the group $G$.

From now on we will assume that the partition of $M$ described in Section 2B is a refinement of its partition into $K$-orbits.

In the following algorithm we use OrbitByPartition (Algorithm 2.3) in two ways: first (with some bookkeeping for the orbit intersection matrices) for the whole orbit $m_{0} G$, the partition being given by the $K$-orbits; and second, the parts being as before, to compute the $K$-orbits.

\section{Algorithm 2.5 (OrbitIntersectionMatrices)}

Input: $m_{0} ; \pi\left(g_{i}\right), 1 \leq i \leq r ; \pi\left(k_{j}\right), 1 \leq j \leq s$

Output:

- a list $L$ containing the minimal elements of $m_{0} G$ with one element of each part marked as a representative

- a map nr: $L \rightarrow\{1, \ldots, m\}$ with $\operatorname{nr}(a)=l$ if $a \in m_{l} K$

- the orbit intersection matrices $A^{(i)} \leftarrow\left(a_{k l}\left(g_{i}\right)\right)$ for $1 \leq i \leq r$

\section{Initialize:}

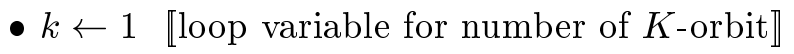

- $L \leftarrow$ OrbitByPartition $\left(m_{0}, \pi\left(k_{1}\right), \ldots, \pi\left(k_{s}\right)\right)$ «start with minimal elements in first $K$-orbit $m_{0} K \rrbracket$

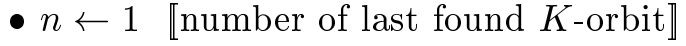

- $\operatorname{nr}(a) \leftarrow 1$ for all $a \in L$

- $A^{(i)} \leftarrow(0)$ for $1 \leq i \leq r$ 【initialize $A^{(i)}$ with $1 \times 1$ zero matrices】

while $k \leq n$ 『loop over the $K$-orbits in $m_{0} G$, evaluating only one of its parts at a time】

$L_{1} \leftarrow$ list of $a \in L$ with $\operatorname{nr}(a)=k$

for each $m^{\prime} \in L_{1}$ marked as a representative of its part

$$
\begin{aligned}
& L_{2} \leftarrow \operatorname{part}\left(m^{\prime}\right) \\
& \text { for } m \text { in } L_{2} \\
& \text { for } i \text { from } 1 \text { to } r \\
& x \leftarrow \operatorname{minimal}\left(m g_{i}\right) \\
& \quad \text { if } x \notin L \llbracket \text { the } K \text {-orbit } x K \text { is not known yet; } \\
& \quad \text { compute it now } \\
& \quad n \leftarrow n+1 \\
& \quad \text { append OrbitByPartition }\left(x, \pi\left(k_{1}\right), \ldots, \pi\left(k_{s}\right)\right) \\
& \quad \text { to } L \\
& \quad \text { et } \operatorname{nr}(a) \leftarrow n \text { for the new points in } L \\
& \quad \text { enlarge all } A^{(j)}, \text { for } 1 \leq j \leq r, \text { by adding } \\
& \quad \text { a column and a row of zeros } \\
& A_{k n}^{(i)} \leftarrow 1 \\
& \text { else } \llbracket \text { number of } K \text {-orbit of } x \text { is known } \rrbracket \\
& l \leftarrow \operatorname{nr}(x) \\
& A_{k l}^{(i)} \leftarrow A_{k l}^{(i)}+1
\end{aligned}
$$

return $L$, nr and $A^{(i)}$ for $1 \leq i \leq r$

Only the minimal points of $m_{0} G$ plus the points of one part at a time have to be stored. Typical orbit intersection matrices are dense. That means that during the execution of this algorithm there are two phases. During the first phase mainly new $K$-orbits 
are evaluated. After the computation of the first few rows of the orbit intersection matrices the list $L$ is complete. In the second phase the remaining part of the orbit intersection matrices is determined.

Remark 2.6. Assume we have run Algorithm 2.5 once and want to know the orbit intersection matrices for additional elements of $G$. This can be achieved by a small modification of the previous algorithm. As input we take the elements of $G$ whose orbit intersection matrices we want to know and the resulting $L$ and nr from a previous call to OrbitlntersectionMatrices. The only difference is now that in the initialization $L$ and $\mathrm{nr}$ are set to the given ones. Of course, here the case $x \notin L$ in the inner loop never occurs.

\section{MATRICES ACTING ON VECTORS}

One case for the setup in Section 2 is that the set $M$ is a finite-dimensional vector (row) space over a finite field and the action of the generators $g_{i}$ (and the $k_{j}$ ) is described by matrices acting on $M$ by right multiplication.

G. Cooperman and M. Tselman [1996] have implemented a parallelized algorithm for computing orbit intersection matrices in this case. An important aspect of their algorithm consists also in saving memory by not storing all points in the orbit. However, if the proportion of stored elements in such an orbit is $1 / \alpha$, one needs on average about $\alpha$ vector-matrix multiplications to find from an arbitrary point in this $K$-orbit one of the stored points. This essentially leads to a multiplication of the total running time of the algorithm by a factor $\alpha$.

Another approach was taken by R. Parker and R. Wilson, who have a (sequential) program that uses "tadpoles" to save memory. There seems to be no reference for this, so here is the idea: One defines a "random-looking" successor function on the set of points and stores only "attracting points" under repeated application of this function. Under certain statistical assumptions one expects to buy a saving factor $1 / \alpha$ in memory usage with a $\log \alpha$ time penalty factor. But it seems to be difficult to predict the behavior of the algorithm in practical cases.

We will now explain a way to realize our functions minimals, minimal and part described in $2 \mathrm{~B}$ efficiently for this case. This allows us to reduce the needed memory by a large factor. But the computing time is only increased by a small constant factor compared to the basic orbit algorithm.

We consider a subgroup $U$ of $K$ with the following properties:

(1) $U$ is small enough that we can store all elements of $U$ in our process for computing the orbit intersection matrices.

(2) There is a $U$-invariant subspace $V$ of $M$ such that all $U$-orbits of the quotient space $M / V$ can easily be computed.

(3) The average length of the $U$-orbits on $M / V$ is "close to" $|U|$.

In practical examples it seems not to be difficult to find such $U$ and $V$. The space $M$ viewed as a $G$-module is typically irreducible. Small subgroups of $K$ as candidates for $U$ can be found by considering some subgroups generated by random elements. Now $M$ considered as $U$-module usually has a composition series consisting of many smalldimensional modules. This can be found using the MeatAxe [Ringe 1998], and so we find candidates for $V$.

Assume that we have found $U$ and $V$ as above. Let pr: $M \rightarrow M / V$ be the projection map. Note that the action of $U$ on $M$ and the induced action on $M / V$ commute with pr. We enumerate $M / V$ and call $m \in M$ minimal if $\operatorname{pr}(m)$ is minimal in its $U$-orbit with respect to this enumeration. ( $p r$ is particularly easy to implement when the basis of $M$ is chosen to contain a basis of $V$.)

In a precomputation - a short one, because of property (2) - we compute by a variation of the basic orbit Algorithm 2.1 for each point of $M / V$ either an element of $U$ mapping it onto the minimal element in its $U$-orbit or, if the point is already minimal, the elements of $U$ stabilizing this point.

Now we implement $\operatorname{part}(m)$ by computing all $m u$, $u \in U$, and removing multiple points. Because of property (3) this takes not much more than one vector-matrix multiplication per element in $\operatorname{part}(m)$.

For minimal $(m)$ we use the precomputation; for $\operatorname{pr}(m)$ we have stored a $u \in U$ such that $m u$ is minimal. All minimals $(m)$ are computed by applying all $u^{\prime}$ from the stabilizer of $\operatorname{pr}(m u)$ to $m u$ and removing multiples. Because of property (3) this stabilizer is often trivial. 
Using these considerations we can count the basic operations needed in Algorithm 2.5.

Proposition 3.1. Assume that in the situation above the computation of minimals $(m)$ takes on average less than 2 vector-matrix multiplications and that the computation of $\operatorname{part}(m)$ takes on average less than 2 vector-matrix multiplications per point in the part. Then:

(a) OrbitByPartition (Algorithm 2.3) requires $2+2 r$ vector-matrix multiplications and $r$ list lookups per point in the considered orbit (neglecting the computation of all minimal points once for each part).

(b) OrbitIntersectionMatrices (Algorithm 2.5) requires $(2+2 s)+(2+2 r)$ vector-matrix multiplications and $s+r$ list lookups per point in the considered orbit (here we neglect the calls to minimals once for each part and the bookkeeping effort for the orbit intersection matrices).

A similar idea also works in the case of $G$ acting on the subspaces of $M$, instead of the vectors.

In certain cases one can think of further improvements by choosing subgroups $U$ with additional nice properties. For example, if $M$ is a semisimple $U$ module then one can take a basis of $M$ such that elements of $U$ have a (very sparse) block diagonal form. (In general one can reach a block triangular form.)

\section{PARALLELIZATION}

We do not see an improvement of Algorithm 2.5 OrbitIntersectionMatrices which reduces the computation time considerably. But we can reduce the waiting time for the result by distributing the computations in parallel among several computer processors. We are mainly thinking of using networks of workstations. In this section we describe our approach to a parallelization of OrbitIntersectionMatrices.

\section{A. Parallel version of OrbitIntersectionMatrices}

Looking at the algorithm we see that it is essential to have a central place where the list $L$ and the map $\mathrm{nr}$ are managed (in form of a hash table) to avoid many computations of the same $K$-orbits by several processes and also to guarantee a unique numbering of the $K$-orbits found.
We divide the work into pieces by giving single runs through a $K$-orbit as jobs to single processors. In such a job, corresponding to a run through the body of the outer loop in Algorithm 2.5, one row of each of the orbit intersection matrices is computed.

We have written a small library which allows communication of processes running on computers connected via a network (using UNIX domain sockets, which are available on many computer operating systems). The communication is of the type that one process sends to another a number indicating a type of a request plus some data. The other process may do some computation and then sends back an answer in form of a block of data. Using this we have implemented three different programs which work together.

First there is one process called the jobserver: It can be asked for a job to do (a number $k$ in Algorithm 2.5), or for a number for a newly found $K$ orbit, and it collects the computed rows of the orbit intersection matrices and stores them into files.

Then there is one process (or several, see below) called the hashserver: This one manages the hash table for the list $L$. It can be asked to send for a given list of points the corresponding numbers of their $K$-orbits or the information which points are lying in a not yet known $K$-orbit. Also this process can be asked to store the information about a new $K$-orbit in its hash table (it writes it to a file, too), and also to send a list of representatives for the parts which are contained in the $K$-orbit with a given number.

Finally there can be many processes called $\boldsymbol{d} \boldsymbol{c}$ client: They ask the jobserver for a job, get the representatives for the $K$-orbit they have to handle from the hashserver, then run through the body of the outer loop of Algorithm 2.5, send the computed rows of the orbit intersection matrices to the jobserver, and start from the beginning. When such a process has to check whether a point is contained in $L$ and wants to know the number of its $K$-orbit then it sends the point to the hashserver to get the answer. When a new $K$-orbit is computed it is sent to the hashserver (which ignores it in the rare case that this $K$-orbit was in the meanwhile already computed by another process). Actually a dcclient does not send single points as requests to the hashserver but always computes minimal $\left(m g_{i}\right)$ for all $m$ in a 
fixed part and puts a collected request to the hashserver into a queue. Before computing the next such request it checks for available answers from the hashserver. This way the client process does not have to be idle in case of a temporarily overloaded network.

As a variant we also allow multiple hashservers: Here we use a function which computes for a given point the number of a hashserver which is responsible to store this point and to answer requests about it. This makes the preparation of hashserver requests by a dcclient slightly more complicated but it can be very useful in certain situations: For example if the data for the requests are computed so fast that the network bandwidth is too small and if we have a switched network (which allows several parallel connections with full bandwidth) then multiple hashservers can increase the overall available network bandwidth for the requests. And this is similar when a hashserver cannot handle all the requests fast enough. Another point is that the hashserver is usually the process which needs most of the memory. For efficiency it is desirable that a hashserver can keep the list $L$ in the physical memory of the computer. Using multiple hashservers we can use the physical memory of several computers for this purpose.

Concerning the memory needed by these processes the hash servers need to hold all minimal points of $m_{0} G$ and a client process needs to store at most all minimal elements of a $K$-orbit and the points in one part of the partition. The orbit intersection matrices can also become very big (there may be up to 10000 $K$-orbits, say). But it is never necessary to store more than one row in a client. Once a row is computed it can be stored in a file and is not needed any more. (We only have to append some zeros when we use them, because some new $K$-orbits can be found after finishing a row.)

The computation time for the algorithm scales almost linearly with the number of clients as long as the bandwidth of the communication between the clients and the hash servers or the computing power of the hash servers do not reach their limit. And the amounts of data which have to be transferred can be estimated very well from 3.1. If network bandwidth becomes a problem one can at least speed up linearly the second phase of the algorithm described after Algorithm 2.5: We interrupt the computation after finding all $K$-orbits and start it again in the form of 2.6 with several hash servers who all use the same already computed data.

\section{B. Comments on the Implementation}

Our implementation of the parallel version of OrbitIntersectionMatrices is written as far as possible in a generic way (the programming language is $\mathrm{C}$ ), where we assume almost nothing about how the points of $M$, elements of $G$ and the action are given. To get a program for a special case one has to write a file containing functions for initializing the clients, operation of group elements on points, the functions part, minimal and minimals, and hash functions for the points. This can then be linked easily with the main part of the program.

The part for the client-server communication is a separate small package which can be used for other programs as well. It supports simple blocking requests, i.e., where a process waits for an answer, as well as queues of nonblocking requests.

One advantage of our communication approach seems to be robustness: The crash of any single process involved in a computation does not waste the computing time spent so far. Client processes can be terminated and new ones started up at any time. Of course the whole computation crashes when one of the server processes is terminated for some reason. But we are saving the results which are already obtained into files and this makes it possible to restart the computation almost at the point where it was stopped. This feature is very important for the use of such programs on networks where any single machine can be down at any time for various reasons. The lack of this feature was also the reason that we did not use a (in certain aspects much more sophisticated) communication protocol like MPI [Snir et al. 1998].

The initial revision of our package contains two versions of the programs. One with permutations as group elements for doing the computation described in $5 \mathrm{C}$ and another more general one for matrices acting on vectors over a finite field. In the latter we use some basic functions from the MeatAxe [Ringe 1998]. Since we want to use this program for very large examples we have put some effort in optimizing the vector-matrix arithmetic, e.g., by precomputing certain linear combinations of rows of the operating 
matrices (Parker calls this "greasing") and by using partial row operations for sparse rows.

Our software is freely available under the Gnu Public License; see Electronic Availability at the end of this article.

\section{DIRECT CONDENSATION}

Let $A$ be a finite-dimensional algebra over a field $F$ and let $e=e \cdot e \in A$ an idempotent. The idea of condensation is to get information on $A$-modules $\mathcal{M}$ by studying the $e A e$-modules $\mathcal{M} e$. In particular this is an important tool in computational representation theory. The latter modules can be of much smaller dimension but still encode interesting information on the structure of $\mathcal{M}$, since the map $\mathcal{N} \mapsto \mathcal{M} e$ is an exact functor from the category of $A$-modules to the category of $e A e$-modules.

For more details we refer to [Cooperman et al. 1997] and the references given there. The first reference describing the use of this method in modular representation theory is J. Thackray's thesis [1981].

\section{A. Interpretation of the OrbitIntersectionMatrices}

We want to consider the special case when $A=F G$ is the group algebra of a finite group $G$ over the field $F, e$ is the idempotent $1 /|K| \cdot \sum_{k \in K} k \in F G$ corresponding to the subgroup $K$ of $G$ whose order is not a multiple of the characteristic of $F$, and $\mathcal{M}$ is a permutation module of $F G$. (If $e$ is of this form then $K$ is called the condensation subgroup).

Now we assume that $G$ and $M$ are finite. A permutation representation $G \rightarrow \operatorname{Sym} M$ of $G$ describes a permutation module $\mathcal{M}$ of $F G$. A basis for this module is parameterized by the elements of $M$. Let $x=\sum_{m \in M} a_{m} m \in \mathcal{M}$ and $O$ be a $K$-orbit of $M$. Then for all $m \in O$ the coefficient of $m$ in $x e$ is $1 /|O| \cdot \sum_{m^{\prime} \in O} a_{m^{\prime}}$. This shows that the orbit sums $\chi_{O}:=\sum_{m \in O} m$ for all $K$-orbits in $M$ are a basis of $\mathcal{M}$ e. Furthermore we see how for $g \in G$ the element ege is acting on this basis: for another $K$-orbit $O^{\prime}$ the coefficient of $\chi_{O^{\prime}}$ in $\chi_{O}$ ege is $1 /\left|O^{\prime}\right| \cdot a_{O, O^{\prime}}$ with $a_{O, O^{\prime}}:=\left|\left\{m \in O \mid m g \in O^{\prime}\right\}\right|$.

Clearly $\mathcal{M}$ is a direct sum of the permutation modules on the $G$-orbits in $M$. Our Algorithm 2.5 (OrbitIntersectionMatrices) computes exactly the numbers $a_{O, O^{\prime}}$ for all $K$-orbits in a single $G$-orbit. (Note that the sum of entries in a fixed row or column of an orbit intersection matrix gives the length of the corresponding $K$-orbit.) The method was called $\boldsymbol{d i}$ rect condensation by Parker and Wilson because one only needs to know for a given point $m \in M$ and $g \in G$ its image $m g$ but one does not need to write down in full detail the explicit permutation induced by $g$ on $M$.

\section{B. An Application with $G=T h$}

As first example for our program we have checked the computations in [Cooperman et al. 1997]. There $G$ is the sporadic simple Thompson group acting linearly on a vector space $M$ of dimension 248 over the field with 2 elements. The considered $G$-orbit has about $10^{9}$ elements. The cited paper contains enough details that we could redo the computations starting with the matrices for this representation given in R. Wilson's WWW-Atlas of group representations [Wilson et al. 1996+].

We used the approach described in Section 3. As subgroup $U$ for the partition of the orbit we constructed a group of order 336 which has an invariant subspace in $M$ of codimension 20 . It turned out that about 1 out of 257 points in the considered $G$ orbit is minimal. The minimal vectors can be stored in 125 Megabytes of memory using one bit per field element.

After we measured the time needed for a single vector-matrix multiplication, we estimated the total running time of the condensation using Proposition 3.1. We found that this estimate was very close to the actual running time. The computations were done on 18 machines $(450 \mathrm{MHz}$ Pentium II processors) of a cluster at the university of St. Andrews (provided by an EPSRC grant), which are connected by a "switched fast ethernet network". We used one hashserver which had to handle about 65 Gigabytes of lookup requests. The computation needed less than 4 hours. (To compare with [Cooperman et al. 1997, 3.3]: There 8 machines computed for one month - a single vector-matrix multiplication took about the same time as in our case - and 610 Megabytes of vectors had to be stored.)

We have also done some larger computations for other sporadic simple groups. The results can hopefully contribute to the determination of the modular character tables of these groups. Details will be given elsewhere. 


\section{C. An Application with $G=S_{21}$ a Symmetric Group}

As another application we condensed the permutation module of a Young subgroup of type $(8,8,4,1)$ in the symmetric group $G=S_{21}$. The motivation was a question by G. James and A. Mathas who could determine the decomposition matrix for the irreducible representations of $G$ in characteristic 5 up to a single entry. The question was whether in the Specht module of $G$ labelled by the partition $(8,8,4,1)$ reduced modulo 5 the irreducible module labelled by $(12,9)$ occurs once or twice.

J. Müller has found a subgroup $K$ of $G$ with the property that the permutation module of type $(8$, $8,4,1)$ condensed with $K$ as condensation subgroup has either 761 or 762 constituents in a composition series depending on the two possible cases. Such a consideration can be made using only the two possible tables of Brauer characters for $G$ and the character table of $K$. (See again [Cooperman et al. 1997] for a more detailed explanation.) The group $K$ is a transitive subgroup of order $47,029,248$ having the number 147 in the database of transitive groups contained in GAP [GAP 2000].

In this case our $M$ consists of 21-tuples of numbers on which $G$ acts by permutation. The permutation module we want to condense is described by the orbit of $[0,0,0,0,0,0,0,0,1,1,1,1,1,1,1,1,2,2,2,2,3]$. It has dimension $1,309,458,150$. As generators of $G$ we took two random elements and $K$ was also given by two generating permutations. As partition of the orbit for Algorithm 2.5 we took again orbits under a subgroup of $K$. There is a nice subgroup which is a direct product of 7 symmetric groups $S_{3}$, each factor permuting the entries of 3 consecutive positions. We define for each part a unique minimal point, namely that one whose entries are sorted in positions $\{1,2,3\},\{4,5,6\}$ and so on. There are only about 660,000 minimal points in the orbit. So, the operation of group elements on the points and the functions part, minimal and minimals are implemented easily and efficiently for this case.

In this example the operation of group elements on points can be computed so fast that even in a parallel computation with few clients the network bandwidth can be reached with the requests for the hashserver. Our approach with multiple hashservers improves the situation considerably here.
On 20 machines of the network already mentioned in 5B using 20 client and 20 hash server processes the orbit intersection matrices for the two generators of $G$ could be computed in 38 minutes and during this time about 96 Gigabytes of data were sent over the network.

The condensed module has dimension 4197. A composition series of this module for the algebra generated by the two computed elements of form ege can be found with the MeatAxe [Ringe 1998] within a few hours of computation time. We found 761 constituents and this rules out the possibility for the decomposition number which would imply 762 constituents. (Note that it is not clear whether our two elements ege generate the whole algebra $e F G e$, but taking further generators into account cannot increase the number of constituents.)

Proposition 5.1. Let $G=S_{21}$ and $F$ be a field of characteristic 5. The multiplicity of the simple $F G$ module labeled by the partition $(12,9)$ in the $F G$ Specht module labeled by $(8,8,4,1)$ is one.

This result together with work of A. Mathas and G. James, in particular the software package Specht [Mathas 1997], determine the decomposition numbers in characteristic 5 for all symmetric groups $S_{n}$ with $n \leq 23$.

\section{ACKNOWLEDGEMENTS}

We would like to thank J. Müller for very useful discussions on the topic.

The cluster of machines used to perform the calculations described in Section 5B was purchased with funds from EPSRC grant GR/M32351.

\section{ELECTRONIC AVAILABILITY}

An implementation of the algorithms described here is freely available under the terms of the Gnu Public License, at http://www.math.rwth-aachen.de/ DC. For details on the Gnu Public License scheme, see http://www.gnu.org/copyleft/gpl.html.

\section{REFERENCES}

[Cooperman and Tselman 1996] G. Cooperman and M. Tselman, "New sequential and parallel algorithms for generating high dimension Hecke algebras using the condensation technique", pp. $155-160$ in 
ISSAC'96: Proceedings of the International Symposium on Symbolic and Algebraic Computation (Zurich, 1996), edited by Y. N. Lakshman, ACM Press, New York, 1996.

[Cooperman et al. 1997] G. Cooperman, G. Hiss, K. Lux, and J. Müller, "The Brauer tree of the principal 19-block of the sporadic simple Thompson group", Experiment. Math. 6:4 (1997), 293-300.

[GAP 2000] The GAP group, GAP: Groups, algorithms, and programming, Version 4.2, RWTH Aachen and University of St. Andrews, 2000. See http:// www-gap.dcs.st-and.ac.uk/ gap.

[Knuth 1997] D. E. Knuth, The art of computer programming, v. 3: Sorting and searching, 2nd ed., Addison Wesley, Reading, MA, 1997.
[Mathas 1997] A. Mathas, Specht: Decomposition matrices for the Hecke algebras of type $A$ (manual for version 2.4), University of Sydney, 1997. See http:// www.maths.usyd.edu.au:8000/u/mathas/specht/.

[Ringe 1998] M. Ringe, The C-MeatAxe, a manual, Lehrstuhl D für Mathematik, RWTH Aachen, 1998. See http://www.math.rwth-aachen.de/ MTX/.

[Snir et al. 1998] M. Snir et al., MPI-the complete reference, 2nd ed., MIT Press, Cambridge (MA), 1998. See also http://www-unix.mcs.anl.gov/mpi/.

[Thackray 1981] J. G. Thackray, Modular representations of finite groups, Ph.D. thesis, Cambridge University, 1981.

[Wilson et al. 1996+] R. Wilson et al., "WWWAtlas of group representations", 1996+. See http:// www.mat.bham.ac.uk/atlas/.

Frank Lübeck, Lehrstuhl D für Mathematik, RWTH Aachen, Templergraben 64, 52062 Aachen, Germany (Frank.Luebeck@math.rwth-aachen.de, http://www.math.rwth-aachen.de/ Frank.Luebeck)

Max Neunhöffer, Lehrstuhl D für Mathematik, RWTH Aachen, Templergraben 64, 52062 Aachen, Germany (Max.Neunhoeffer@math.rwth-aachen.de, http://www.math.rwth-aachen.de/ Max.Neunhoeffer)

Received May 10, 2000; accepted in revised form October 13, 2000 